\title{
An alternative approach to the relation between accretion luminosity and mass accretion rate
}

Stefano Pezzuto ( $\square$ stefano.pezzuto@inaf.it)

Institute for Space Astrophysics and Planetology

\section{Research Article}

Keywords: accretion, lacc, mass, luminosity, rate, matter

Posted Date: August 3rd, 2021

DOI: https://doi.org/10.21203/rs.3.rs-770994/v1

License: (c) (i) This work is licensed under a Creative Commons Attribution 4.0 International License. Read Full License 


\title{
An alternative approach to the relation between accretion luminosity and mass accretion rate
}

\author{
Stefano Pezzuto ${ }^{1, *}$ \\ ${ }^{1}$ INAF-IAPS, via Fosso del Cavaliere, 100, I-00133, Roma, Italy \\ *stefano.pezzuto@inaf.it
}

\begin{abstract}
In this paper I introduce and discuss an alternative approach to the relation between accretion luminosity, $L_{\text {acc }}$, and mass accretion rate, $\dot{M}$ : instead of the universally adopted $L_{\mathrm{acc}}=G M \dot{M} / R$, I propose the dynamical definition $L_{\mathrm{acc}}=v_{\mathrm{f}}^{2} \dot{M} / 2$ where $v_{\mathrm{f}}$ is the final velocity of the infalling matter at the surface of the accreting object of mass $M$ and radius $R$. Both definitions are based on the energy conservation, but while the former assumes that matter is in free fall, the latter is valid always. By adopting the alternative form for $L_{\mathrm{acc}}$, I show that the Eddington luminosity $L_{\mathrm{ed}}$, when the outward radiation pressure wins on gravity, is never produced with a finite $\dot{M}$. Instead, $L_{\text {ed }}$ is a limit asymptotically reached when $\dot{M} \rightarrow \infty$. My argument is very simple, so I felt the need to find a possible explanation to why no one arrived to this conclusion before. To this aim, I give a brief presentation of the history of accretion, from the pioneer work of Hoyle and collaborators until the '60s of last century, to show how the perception of the role of the radiation pressure in accretion evolved. I give also some practical applications of the formulae I derived, in the case of high-mass star formation and of the growth of super massive black holes. The study of these two processes, already complex per se, becomes more difficult to solve because of the existence of a limiting $\dot{M}$, named Eddington mass accretion rate or $\dot{M}_{\text {ed }}$, that it is supposed to generate a luminosity equal to $L_{\text {ed }}$, making it impossible to accrete at rate $\dot{M}>\dot{M}_{\text {ed }}$. Accretion rates higher than $\dot{M}_{\text {ed }}$ are however necessary, as theory and observations show. My definition of $L_{\text {acc }}$ takes naturally into account the work done by radiation pressure to slow down the infalling matter: as a consequence, $L_{\text {acc }}$ does not increase linearly with $\dot{M}$ and $L_{\text {ed }}$ is only an asymptotic value.
\end{abstract}

\section{Introduction}

In this introduction I present some basic facts on accretion. I avoided to add references as much as possible because what I write here can be found in any paper on acretion. A fundamental text on this argument is the book by ${ }^{1}$.

Accretion is an important process in astrophysics: energy can be extracted from matter with an efficiency that, in compact objects, is even greater than that available in nuclear reactions. This is because the efficiency of the process depends on the mass to radius ratio $M / R$ of the accreting object, which can be very high for neutron stars. In the case of star formation $M / R$ is smaller by order of magnitudes but accretion is anyway important not only because the mass of a star eventually arises from the matter falling on the core, but also because during the early phases of the star formation, accretion is the only mechanism that produces energy.

The amount of energy that can be radiated per unit time by accretion, $L_{\mathrm{acc}}$, as well as how much of that energy arrives to the observer, depend on many factors: the geometry (spherical vs. disk accretion), the composition of infalling matter (dust grains instead of neutral or ionized gas), the nature of the accreting object (in particular if it has a surface or it does not, like a black hole), the presence of a magnetic field.

If the precise value of $L_{\mathrm{acc}}$ depends on the particolar accretion scenario, there is a general consensus that the maximum possible value for a given mass accretion rate $\dot{M}$ is

$$
L_{\mathrm{acc}}=\frac{G M \dot{M}}{R}
$$

which assumes that all the kinetic energy of the infalling matter is thermalized and radiated away once the surface of the accreting object is hit.

When $L_{\text {acc }}$ is high enough, radiation pressure starts influencing the motion of the infalling matter. In particular, the radiation transfers outward momentum to the matter that is slowed down; when this pressure equals the gravitational force accretion is stopped or even reverted. The luminosity that can stop accretion is named $L_{\text {ed }}$ after Eddington, who found that for massive stars the radiation pressure due to the thermonuclear reactions can make the star atmosphere evaporate.

Given the linear relation between $L_{\text {acc }}$ and $\dot{M}$ in Eq. (1), it is immediate to derive the $\dot{M}$ at which $L_{\text {acc }}=L_{\text {ed }}$. This $\dot{M}$ is also generally named $\dot{M}_{\text {ed }}$ after Eddington. 
The fact that $L_{\mathrm{ed}}$ can be reached with a finite value of $\dot{M}$ has important implications in astrophysics, for instance in topics like high-mass star formation, accretion onto compact objects or growth of super massive black holes. In these cases, the observational results sometimes require to model the accretion with high rates, such that $\dot{M}>\dot{M}_{\text {ed }}$.

Motivated by the well-known effect of the radiation pressure that slows down the motion of the infalling particles, in this paper I investigate the consequences of adopting an alternative point of view in deriving $L_{\text {acc }}$, namely

$$
L_{\mathrm{acc}}=\frac{1}{2} v_{\mathrm{f}}^{2} \dot{M}
$$

where $v_{\mathrm{f}}$ is the final velocity that matter has when reaching the surface of the accreting object. This relation is based on the energy conservation, exactly like Eq. (1), with the important difference that while Eq. (1) assumes that $v_{\mathrm{f}}$ is the velocity of matter in free fall, Eq. (2) does not make any a-priori assumption on the value of $v_{\mathrm{f}}$ : this is to be derived from the specific accretion model used.

To show the consequences of adopting Eq. (2) instead of Eq. (1) I use a simple, spherically-simmetric accretion model of ionized hydrogen, whose equation of motion can be easily solved analitycally. I found that the radiation pressure cannot stop accretion because $L_{\text {acc }}<L_{\text {ed }}$ for any finite $\dot{M}$, even for $\dot{M}>\dot{M}_{\text {ed }} . L_{\text {acc }}$ provides a negative feedback to the accretion, through the radiation pressure, causing a self-regulation of the process, limiting $L_{\text {acc }}$ to remain below $L_{\text {ed }}$ for any value of $\dot{M}$.

Before concluding this Introduction, I wish to stress few important points: first, this paper is not aimed at presenting a new accretion model. The model I use here was already developed and studied in the 70's of the last century. The spherical accretion model itself can be dated back to the work by ${ }^{2}$. I use this model only as an example to show what happens using Eq. (2) instead of Eq. (1). The aim of this paper, in fact, is to promote the use of Eq. (2) when radiation pressure is important; $L_{\text {acc }}$ should always be an outcome of the model once $v_{\mathrm{f}}$ is derived, instead of being assumed a-priori from Eq. (1). Finally, cases where from observations it turns out that $L_{\mathrm{acc}}>L_{\mathrm{ed}}$ are not considered here because, in the model adopted for this paper, they cannot be accounted for: no matter how high $\dot{M}$ is, it will always be that $L_{\text {acc }}<L_{\text {ed }}$.

The paper is organized in this way: in Section $2 L_{\mathrm{ed}}$ and $\dot{M}_{\mathrm{ed}}$ are derived in the case Eq. (1) is considered valid; then I show what are the consequences of adopting Eq. (2) instead. First the case of an accreting object without an internal source of luminosity is presented $\left(L_{\star}=0\right)$, then the cases of $L_{\star} \neq 0$ and of black holes are considered. In Section 3 I make a comparison between my results and those presented in literature. This section gives a basic overview of the literature on accretion and is not meant to be, nor it could be, complete. I also give a short historical review on accretion with emphasis on the role of the radiation pressure. In Section 4 some pratical examples are given in the case of high-mass star formation, in particular on interpreting the burst of mass accretion rate that is observed in some high-mass forming stars; and in the case of the growth of super massive black holes. Conclusions are in Section 5.

\section{The Eddington limit on luminosity and its relation with the mass accretion rate}

Consider an object of mass $M$ and radius $R$ which accretes matter under the following assumptions: accretion is steady and spherically symmetric with no angular momentum; infalling material is fully ionized hydrogen whose opacity is Thomson scattering; the infalling flow is at zero temperature. This last hypothesis means that falling particles do not interact each other on their way to the star, except for the interaction electron-proton. The object emits radiation at a rate $L$ that, for the moment, we assume is due to accretion only so that $L \equiv L_{\text {acc }}$.

The radiation pressure due to Thomson scattering acts like a force that transfers momentum to matter. In the classical treatment of radiation ${ }^{3}$ this momentum is completely absorbed by matter at a rate $W \sigma$ where $W$ is the energy density of the electromagnetic field and $\sigma$ is the Thomson cross section. Since $\sigma$ depends on the inverse of the square of the particle mass, this force acts mainly on the electrons. On the contrary, the gravitational force is larger for protons: as a consequence the flow should be treated as a two fluids plasma but the hypothesis of fully ionized hydrogen allows us to treat the problem as one single fluid. In fact, the electric field arising from the interactions between protons and electrons keeps the two components coupled $^{4}$. The total force acting on each particle is then

$$
F_{\mathrm{tot}}=\frac{\sigma I}{c}-\frac{G M m}{r^{2}}
$$

$m=1.7 \times 10^{-24} \mathrm{~g}$ being the proton mass and $\sigma=6.7 \times 10^{-25} \mathrm{~cm}^{2}$ the Thomson cross section for electrons. $I=c W$ is the intensity field.

If absorption is negligible then $I$ scales as $L_{\mathrm{acc}} r^{-2}$ so that

$$
F_{\mathrm{tot}}=\frac{\sigma L_{\mathrm{acc}}}{4 \pi c r^{2}}-\frac{G M m}{r^{2}} .
$$


The Eddington luminosity $L_{\mathrm{ed}}$ is the value of $L_{\mathrm{acc}}$ in the above equation that makes $F_{\mathrm{tot}}=0$, i.e.

$$
L_{\mathrm{ed}} \equiv \frac{4 \pi c m}{\sigma} \cdot G M=3.3 \times 10^{4}\left(\frac{M}{M_{\odot}}\right) L_{\odot} .
$$

From Eq. (1) the limit on luminosity is immediately turned into a limit on the accretion rate

$$
\dot{M}_{\mathrm{ed}} \equiv \frac{4 \pi c m R}{\sigma}=1.1 \times 10^{-3}\left(\frac{R}{R_{\odot}}\right) \dot{M}_{\odot} / \mathrm{yr} .
$$

If a star accretes at $\dot{M}_{\text {ed }}$ the radiation pressure stops accretion because of Eq. (4), from which it is concluded that it is impossible to accrete at a rate $\dot{M}>\dot{M}_{\text {ed }}$. This demonstration can be found in the classical book on accretion by ${ }^{1}$; it was presented also $\mathrm{in}^{5}$. Note that this demonstration is based on two equations: first $L_{\mathrm{ed}}$ is derived from Eq. (4). Then, Eq. (1) is used to show that $L_{\mathrm{ed}}$ can be attained with a finite $\dot{M}$. These two equations are independent each other: actually, what is worse, they are not self-consistent.

In fact, when $\dot{M}=\dot{M}_{\text {ed }}$ the two equations do not admit a common solution: Eq. (1) predicts $L_{\text {acc }}=L_{\mathrm{ed}}$ so that in Eq. (4) $F_{\text {tot }}=0$, i.e., accretion is halted; but if accretion is halted $L_{\mathrm{acc}}=0$. One might conclude that under this condition the flow toggles between active/halted states. Another possibility could be that for $\dot{M}=\dot{M}_{\text {ed }}$ the approximation of zero-temperature stops being valid and the gas pressure should enter the game making time-dependant solutions be possible, like those already found by ${ }^{6}$. In any case, the above demonstration is not adequate to describe the condition $\dot{M}=\dot{M}_{\text {ed }}$, which in turn makes questionable to conclude that we have demonstrated that $L_{\mathrm{ed}}$ is produced when $\dot{M}=\dot{M}_{\mathrm{ed}}$.

Let us now look at accretion by considering the problem from the point of view of energy conservation. When the infalling of matter happens at very low $\dot{M}$, radiation pressure can be neglected. A particle at rest at distance $r$ from a star of mass $M$ feels an attraction

$$
F \equiv m \frac{\mathrm{d} v}{\mathrm{~d} r}=-\frac{G M m}{r^{2}}
$$

or

$$
m \mathrm{~d} v=-\frac{G M m}{r^{2}} \frac{\mathrm{d} r}{v}
$$

which integrated gives

$$
\frac{1}{2} v^{2}=\frac{G M}{r}
$$

Once the particle hits the surface of the star of radius $R$ its kinetic energy is

$$
\frac{1}{2} m v_{\mathrm{f}}^{2}=\frac{G M m}{R}
$$

where $v_{\mathrm{f}}$ is the final velocity. So, Eq. (1) is the consequence of energy conservation for a particle in free-fall motion, along with the hypothesis that $100 \%$ of the kinetic energy can be converted in radiation.

But if the radiation pressure slows down the infalling matter, we are not in free fall anymore. The equation of motion is now given by Eq. (4). In turn, this means that the final velocity cannot be given by Eq. (10) on which Eq. (1) is based. Radiation is doing work on the matter, so that the energy balance of the system cannot be the one expected for free-fall motion.

If we start from Eq. (4), Eq. (8) becomes

$$
m v \mathrm{~d} v=\left[\frac{\sigma L_{\mathrm{acc}}}{4 \pi c r^{2}}-\frac{G M m}{r^{2}}\right] \mathrm{d} r
$$

that, after an easy integration, brings to the equivalent of Eq. (10) for the case where radiation pressure is not negligible:

$$
\frac{1}{2} m v_{\mathrm{f}}^{2}=\frac{G M m}{R}-\frac{\sigma L_{\mathrm{acc}}}{4 \pi c R} .
$$

We now make use of Eq. (2) to eliminate $v_{\mathrm{f}}$ finally obtaining

$$
L_{\mathrm{acc}}=\frac{G M \dot{M}}{R} \cdot \frac{1}{1+\mu}
$$


or, equivalently,

$$
L_{\mathrm{acc}}=L_{\mathrm{ed}} \cdot \frac{\mu}{1+\mu}
$$

where $\mu \equiv \dot{M} / \dot{M}_{\text {ed }}$.

For the final velocity we find from Eq. (12)

$$
v_{\mathrm{f}}=v_{\mathrm{ff}}\left(1-\frac{\mu}{1+\mu}\right)^{1 / 2}=\frac{v_{\mathrm{ff}}}{\sqrt{1+\mu}}
$$

where $v_{\mathrm{ff}}$ is the free-fall velocity at $r=R$.

These equations are valid for any given $\dot{M}$, also when $\mu>1$, i.e. $\dot{M}>\dot{M}_{\text {ed }}$. In particular

$$
L_{\mathrm{acc}}\left(\dot{M}_{\mathrm{ed}}\right)=\frac{1}{2} L_{\mathrm{ed}}
$$

which means that $\dot{M}_{\text {ed }}$ is just the mass accretion rate at which the accretion luminosity is one half of the largest possible value $L_{\text {ed }}$, neither it is an upper limit to $\dot{M}$ nor it produces a luminosity corresponding to $L_{\mathrm{ed}}$.

When $\dot{M} \ll \dot{M}_{\text {ed }}$ the second term of Eq. (13) tends to unity and Eq. (1) is recovered: in this regime $L_{\text {acc }}$ is directly proportional to $\dot{M}$. At accretion rates such that $\dot{M} \gg \dot{M}_{\text {ed }}$ Eq. (14) tells us that the luminosity no longer increases proportionally to $\dot{M}$ but gets closer and closer to the value $L_{\text {ed }}$ given by Eq. (5), asymptotically reached when $\dot{M} \rightarrow \infty$.

Equation (13) can be written also as

$$
L_{\mathrm{acc}}=\frac{G M \dot{M}_{\mathrm{eff}}}{R}
$$

where $\dot{M}_{\text {eff }} \equiv \dot{M} /(1+\mu)$ is the effective mass accretion rate: in the limit $\mu \rightarrow \infty, \dot{M}_{\text {eff }} \rightarrow \dot{M}_{\text {ed }}$ and Eq. (1) can be written as $L_{\text {ed }}=G M \dot{M}_{\text {ed }} / R$. Clearly, the equivalence between Eq. (1) and the asymptotic form of Eq. (16) is only formal.

We can now understand better what Eqs. (5) and (6) mean. $L_{\text {ed }}$ depends only on the mass, so that it is the same for the Sun, or for a $1 M_{\odot}$ neutron star. This depends on the implicit assumption, hidden in Eqs. (1) and (7), that the central source has an omogeneous and uniform density, so that the gravitational force depends on the distance $r$ from the centre, and on the total mass $M$. The radius $R$ of the star, for $r>R$, does not play any role. $L_{\text {ed }}$ is the maximum luminosity that can be generated by accretion onto a point-source of mass $M$. That's why $L_{\mathrm{ed}}$ is the same for the Sun, for a neutron star or for a black hole of $3 \mathrm{~km}$. And this consideration gives also an intuitive reason on why $L_{\text {ed }}$ can never be emitted, whatever $\dot{M}$ is: only a point-source $(R=0)$ mass could potentially reach that luminosity.

On the other hand, $\dot{M}_{\text {ed }}$ depends on the radius only: for the Sun it is $1.1 \times 10^{-3} M_{\odot} \mathrm{yr}^{-1}$, but for a neutron star with $R=10 \mathrm{~km}, \dot{M}_{\text {ed }}$ is only $1.5 \times 10^{-8} M_{\odot} \mathrm{yr}^{-1}$. The radius is the point where the falling motion is stopped and the radiation is produced. The smaller $R$, the higher the kinetic energy. As we have seen before, $L_{\mathrm{ed}}$, the highest accretion luminosity, is reached when $\dot{M} \gg \dot{M}_{\text {ed }}$, so $\dot{M}_{\text {ed }}$, or better $\mu$, is a measure, as long as accretion luminosity is concerned, of the compactness of the accreting source: when $R$ decreases the central object is more similar to a point-source, so that it is necessary a smaller $\dot{M}$ to cause $\mu>1$ and $L_{\mathrm{acc}}$ being closer and closer to $L_{\mathrm{ed}}$. In the limit case of $R=0, \dot{M}_{\mathrm{ed}}=0$ and $\mu \rightarrow \infty$ so that $L_{\mathrm{acc}}=L_{\mathrm{ed}}$, from Eq. (14): any $\dot{M}$ will produce a luminosity $L_{\mathrm{ed}}$. From this point of view, Eddington mass accretion rate is not an appropriate name for this particular $\dot{M}$; however, I will keep using $\dot{M}_{\text {ed }}$ for consistency with previous works.

The case $L_{\star} \neq 0$

If the accreting object has an internal source of energy generating a luminosity $L_{\star}$ independent on accretion, there are two possibilities. If $L_{\star}>L_{\text {ed }}$ then accretion is not possible unless the ram pressure of the infalling matter is taken into account ${ }^{7,8}$. When ${ }^{9} M_{\star} \gtrsim 13 M_{\odot}$ then $L_{\star}>L_{\mathrm{ed}}$. This is because the presence of the dust changes the opacity to values as high as ${ }^{10}$ $8 \mathrm{~cm}^{2} \mathrm{~g}^{-1}$, and $L_{\mathrm{ed}}=1600\left(M / M_{\odot}\right) L_{\odot}$.

On the other hand, when $L_{\star}<L_{\mathrm{ed}}$ the previous equations are still correct if $L$ in Eq. (12) is interpreted as the sum of $L_{\star}+L_{\text {acc}}$. Eqn. (2) gives the relation between $L_{\mathrm{acc}}$ and $v_{\mathrm{f}}$ and finally Eq. (14) becomes

$$
L_{\mathrm{acc}}=\left(L_{\mathrm{ed}}-L_{\star}\right) \frac{\mu}{1+\mu}
$$

If $\dot{M}=0$ clearly $L_{\text {acc }}=0$; as $\dot{M} \rightarrow \infty$ then $L_{\text {acc }} \rightarrow\left(L_{\text {ed }}-L_{\star}\right)$. Let's see the implications for the total luminosity of the system.

Adopting Eq. (1) we have $L_{\mathrm{tot}}=L_{\star}+L_{\mathrm{acc}}=L_{\star}+G M \dot{M} / R$; by defining $\Lambda=L_{\mathrm{tot}} / L_{\mathrm{ed}}$ and $\Lambda_{\star}=L_{\star} / L_{\mathrm{ed}}$ we get $\Lambda=\Lambda_{\star}+\mu$. Mathematically, it is trivial to plot $\Lambda$ as a function of $\mu$ : this is shown in top panel of Fig. 1 in the range $0.1 \leq \mu \leq 10$, for 

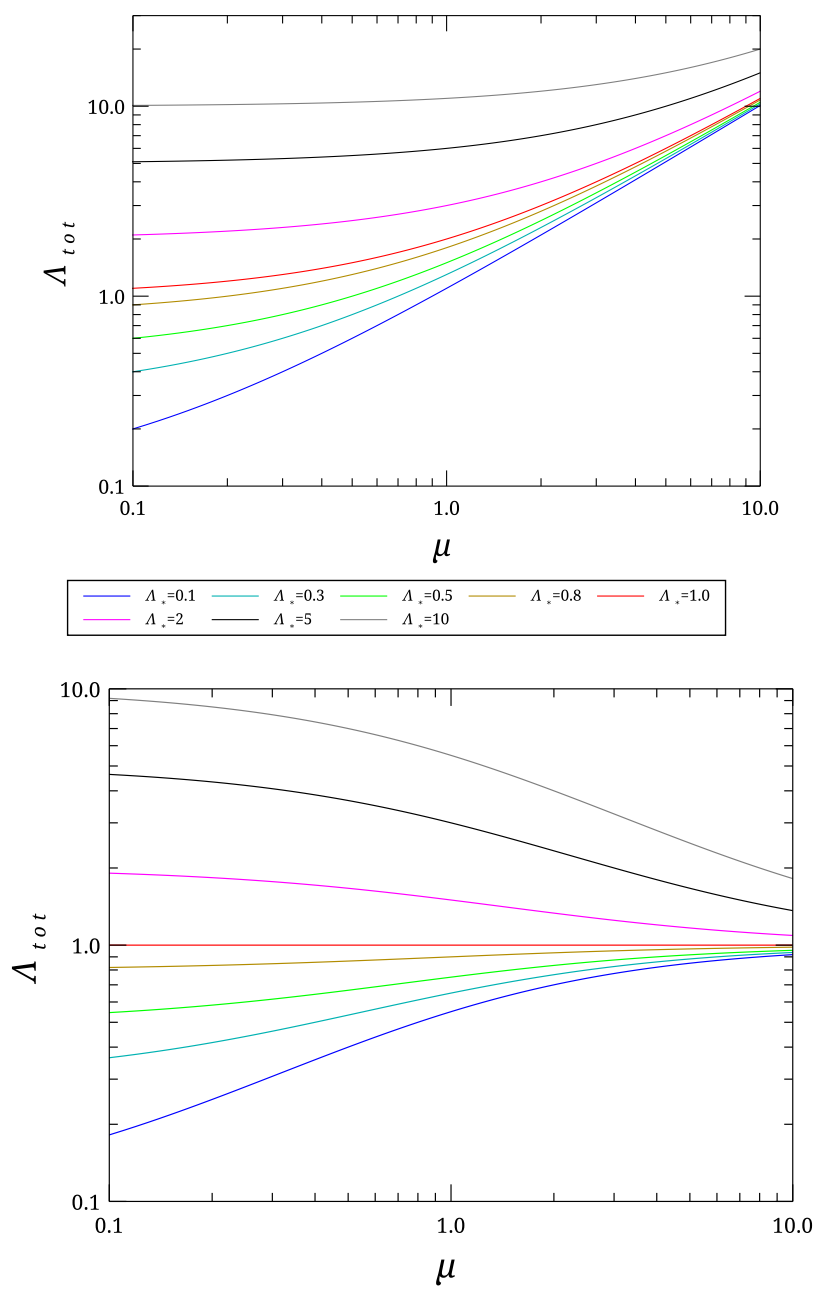

Figure 1. Top panel: total luminosity vs. mass accretion rate when $L_{\mathrm{acc}}=G M \dot{M} / R$ is assumed. Total luminosity and mass accretion rate are parametrized in terms of $L_{\mathrm{ed}}\left(\Lambda=L_{\mathrm{tot}} / L_{\mathrm{ed}}\right)$ and $\dot{M}_{\mathrm{ed}}\left(\mu=\dot{M} / \dot{M}_{\mathrm{ed}}\right)$, respectively. Eight different cases are presented for different star luminosities, also given in units of $L_{\mathrm{ed}}: \Lambda_{\star}=L_{\star} / L_{\mathrm{ed}}$. Bottom panel: same as in top panel, but when the total luminosity is given by Eq. (20).

different values of $\Lambda_{\star}$. Actually, $\Lambda$ vs. $\mu$ is more complex than it seems because accretion is not possible either when $\mu \geq 1$ or $L_{\star}>L_{\text {ed }}$ (then $L_{\text {acc }}=0$ and $L_{\text {tot }}=L_{\star}$ ) even if $\Lambda$ is defined, at least mathematically, for any value of $\Lambda_{\star}$ and $\dot{M}$ and then for any value of $\mu$. In other words, we have to impose by hand that accretion is stopped, because neither from Eq. (1), nor from $L_{\mathrm{tot}}=L_{\star}+G M \dot{M} / R$ can we derive this condition.

If, on the contrary, we start from Eq. (17), then

$$
L_{\mathrm{tot}}=L_{\star}+L_{\mathrm{acc}}=L_{\star}+\left(L_{\mathrm{ed}}-L_{\star}\right) \frac{\mu}{1+\mu}=\frac{L_{\star}+\mu L_{\mathrm{ed}}}{1+\mu}
$$

or, equivalently

$$
\Lambda=\frac{\Lambda_{\star}+\mu}{1+\mu} .
$$

The product $\mu L_{\mathrm{ed}}$ is nothing but $G M \dot{M} / R$ so that Eq. (18) for $L_{\mathrm{tot}}$ is similar to Eq. (13) for $L_{\mathrm{acc}}$

$$
L_{\mathrm{tot}}=\left(L_{\star}+\frac{G M \dot{M}}{R}\right) \frac{1}{1+\mu} .
$$


The trend of $\Lambda$ with $\mu$ of Eq. (19) is shown in the bottom panel of Fig. 1, for the same set of values of $\Lambda_{\star}$ as in the top panel.

What Eq. (18) is telling us is that when $L_{\star}>L_{\mathrm{ed}}$, the work made by radiation pressure to stop the infalling matter and to revert its direction has the effect that the radiation reaching the observer is less than the original $L_{\star}$, even if $L_{\star}$ itself does not depend on $\dot{M}$ (and, clearly, $L_{\mathrm{acc}}=0$, so it gives no contribution to $L_{\mathrm{tot}}$ ). Higher $\dot{M}$ requires more work to be done so that, when $\mu \gg 1, L_{\text {tot }}$ is more and more reduced and tends asimptotically to $L_{\mathrm{ed}}$. Eq. (18) gives a family of solution $\left(\Lambda_{\star}, \mu\right)$ that describes $L_{\text {tot }}$ in a smooth way without the irregular behaviour of $L_{\text {acc }}$ that is seen when Eq. (1) is adopted.

Another important point to highlight is that the factor $(1+\mu)^{-1}$ in Eq. (20) has a different meaning when applied to the first or to the second term in the right hand side of the equation. The term $L_{\star} /(1+\mu)$ describes how the radiation from the star is reduced for the work done on the infalling matter, but $L_{\star}$ itself does not change. In this sense, it is similar to the idea at the base of RIAF models (see next section): the generated luminosity is constant, but the radiation reaching the observer is somehow attenuated. On the contrary, the term $G M \dot{M} / R(1+\mu)$ means that $L_{\text {acc }}$ is effectively generated at a reduced rate, because matter arrives at the star surface with a lower velocity when $\dot{M}$, and so $\mu$, increases.

In real world, the zero-temperature is a poor hypothesis at high $\dot{M}$ : clearly, the deceleration of the matter will cause a sort of atmosphere around the accreting object that can be modelled only if we consider the interaction between particles, which implies using thermodynamics laws. In any case, the point here is that the work done by radiation pressure is forgotten when one uses blindly Eq. (1).

I will show later two examples of using Eq. (20) in case of burst of accretion. In this case, the equation is valid for both the quiescent phase and during the burst. The only difference is that during the burst phase, $\dot{M}$ is the total mass accretion rate, including also the constant $\dot{M}$ during quiescience, in case it is not zero.

\section{The case of black holes}

For a black hole we can make the hypothesis that matter flows radially under the influence of the gravitational field until it reaches the distance $R \gtrsim R_{\mathrm{S}}=2 G M / c^{2}$, where $R_{\mathrm{S}}$ is the Schwarzschild radius. Then, its kinetic energy is released as radiation energy ${ }^{11}$. For this paper it is not important to specify the physical mechanism that produces the radiation, exactly like in the previous section it was not specified how the kinetic energy of the gas, once hits the surface of the central object, is transformed in thermal energy and then radiated away.

Introducing $R_{\mathrm{s}}$ in Eq. (1) we get

$$
L_{\mathrm{acc}}=\frac{c^{2} \dot{M}}{2}
$$

assuming that $100 \%$ of the kinetic energy is converted into radiation. A multiplicative factor $\eta$ can be put in the right hand side of the equation, as can be done in Eq. (1), usually 0.1 for black holes, but we can set it to 1 for simplicity.

$L_{\text {ed }}$ is still given by Eq. (5), while $\dot{M}_{\text {ed }}$ can be expressed in terms of the mass of the black hole by introducing $R_{\mathrm{S}}$ in Eq. (6)

$$
\dot{M}_{\mathrm{ed}}=\frac{8 \pi m G M}{c \sigma}=4.5 \times 10^{-9}\left(\frac{M}{M_{\odot}}\right) \dot{M}_{\odot} \mathrm{yr}^{-1}
$$

Eq. (13) becomes

$$
L_{\mathrm{acc}}=\frac{c^{2} \dot{M}}{2} \frac{1}{1+\mu}
$$

where, again, $L_{\mathrm{acc}} \rightarrow L_{\mathrm{ed}}$ only for $\dot{M} \rightarrow \infty$. Eq. (14), that gives $L_{\mathrm{acc}}$ as function of $L_{\mathrm{ed}}$, does not change.

\section{Comparison with previous results}

As pointed out by ${ }^{12}$, there are two main different problems in accretion: how much radiation can be produced by accretion, and how much of this radiation actually reaches the observer. For the former question Eq. (1), or Eq. (21) for black holes, have always been the starting point, with at most a corrective factor if the efficiency was assumed less than $100 \%$. This makes it not easy a comparison between the results presented in this work with those found in literature: the relation between $L_{\text {ed }}$ and $\dot{M}_{\text {ed }}$ was given as a matter of fact, it has never be felt the need to question its validity. Thus, the results, or their interpretation, derived with Eq. (17) are different from those found in previous works.

As an example, let us consider Eq. (4): it can be cast in the following alternative form

$$
F_{\text {tot }}=-\frac{G M^{\prime} m}{r^{2}}
$$




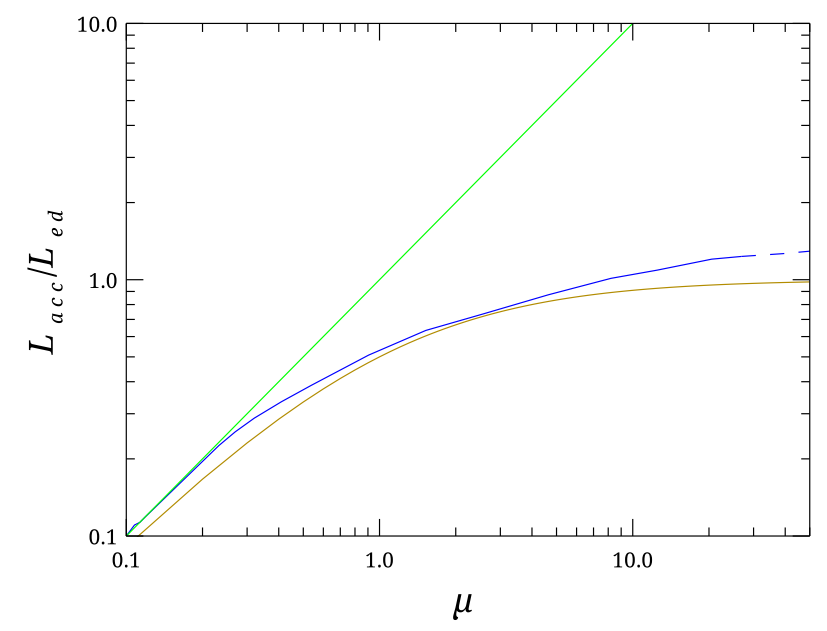

Figure 2. Ratio $L_{\text {acc }} /$ led as a function of $\mu=\dot{M} / \dot{M}_{\text {ed }}$. Green line: linear relation from Eq. (1); blue line: data from ${ }^{16}$; brown line: relation from Eq. (14).

where

$$
M^{\prime}=M-\frac{\sigma L_{\mathrm{acc}}}{4 \pi G c m}=M\left[1-\frac{L_{\mathrm{acc}}}{L_{\mathrm{ed}}}\right]=M \frac{1}{1+\mu}
$$

which means that the effect of the radiation pressure is numerically equivalent to the reduction of the stellar mass. The Eddington luminosity is then the luminosity produced by accretion onto a star such that the effective mass given by Eq. (25) is zero. The correct formula for accretion luminosity should be then

$$
L_{\mathrm{acc}}=\frac{G M^{\prime} \dot{M}}{R} .
$$

The idea that $L_{\text {acc }}$ causes an effective mass $M^{\prime}<M$ as in Eq. (24) was derived by ${ }^{13}$. Nonetheless, the relation $L_{\text {acc }}=G M \dot{M} / R$ was still used and, as a consequence, $L_{\text {acc }} / L_{\text {ed }}=\dot{M} / \dot{M}_{\text {ed }}$; the effective mass was then written $M^{\prime}=M\left(1-\dot{M} / \dot{M}_{\text {ed }}\right)=M(1-\mu)$ instead as in Eq. (25).

The conclusion that the mass accretion rate is not limited to a finite value was already found by ${ }^{14}$. However in their model they assumed that the maximum emergent luminosity is $L_{\mathrm{acc}}=G M \dot{M} / R$ and the accretion flow is not stopped because the power released by accretion does not emerge through the infalling matter. Thus, the radiation pressure is never high, whatever $\dot{M}$ is. This result belongs to a class of models where the basic idea is that very little of the gravitational potential energy of the inflowing gas is radiated (the so-called radiatively inefficient accretion flows or RIAFs, see ${ }^{15}$ and references therein). However the low luminosity of RIAF models is due to inefficiency in the emission of radiation, while the intrinsic luminosity available from accretion is still assumed equal to $G M \dot{M} / R$.

From Eq. (1) it was found by ${ }^{4}$ that "the deceleration produced by radiation pressure does not stop the flow of the gas" which is also my conclusion. However, they introduced the ratio $\xi=L_{\mathrm{acc}} / L_{\mathrm{ed}}$ varied as free parameter up to the value 1: in my treatment $\xi=1$ means $\dot{M} \rightarrow \infty$ while, assuming Eq. (1), it means $\dot{M}=\dot{M}_{\text {ed }}$.

A relation between $L_{\mathrm{acc}}$ and $\dot{M}$ was found by ${ }^{16}$ and reported in Fig. 2 as blue line. The relation given by Eq. (14) of the present work is shown as brown line. The similarity between the two curves is impressive, but, as in the previous case, does not mean that the results are physically comparable. The case studied by ${ }^{16}$ was that of a disk accreted by a black hole assuming Eq. (1). The ratio $L_{\mathrm{acc}} / L_{\mathrm{ed}}$ was found to be upper-bound, in a qualitative agreement with my Eq. (14), but the reason was not the radiation pressure, rather the fact that a fraction of kinetic energy was lost with the matter swallowed by the black hole.

An effective mass defined in terms of luminosity, as in Eq. (25), was introduced also by ${ }^{17}$ but he used Eq. (21) for luminosity, and assuming an efficiency $\eta \neq 1$ he wrote $\dot{M}_{\mathrm{ed}}=L_{\mathrm{ed}} /\left(\eta c^{2}\right)$.

The formation of the first population of stars was investigated by ${ }^{18}$ who introduced an $\dot{M}_{\text {crit }}$ above which the total luminosity of a star $L_{\text {tot }}=L_{\star}+L_{\text {acc }}>L_{\text {ed }}$. But according to Eq. (17) if $L_{\star}<L_{\text {ed }}$, then $L_{\star} \leq L_{\text {tot }}<L_{\text {ed }}$ : no matter how large $\dot{M}$ is, the total luminosity will never be larger than $L_{\mathrm{ed}}$. 
Reasons why it is not correct to conclude that an accretion rate $\dot{M}>\dot{M}_{\text {ed }}$ should stop accretion were reviewed by ${ }^{19}$. However, he did not question Eq. (21), instead, he discussed which conditions can strengthen the gravity field, like rotation of the central source, or can weaken the radiative force. Further, he was mainly discussing cases where $L_{\mathrm{acc}}>L_{\mathrm{ed}}$ that are not covered in my work.

Instead of continuing with other comparisons with previous results, that would add nothing more to what already written, it is much more interesting to address the problem of why Eq. (1) was never questioned in the past. To shed some light on this problem, we have to look back at accretion in an historical perspective.

First of all, the luminosity we are dealing with had nothing to do with accretion. It was derived by ${ }^{20}$ when studying the internal equilibrium of stars (actually, three years before this work the importance of radiation pressure was already pointed out by Białobrzeski, as reported by ${ }^{19}$; Eddington discovered Białobrzeski's result after the end of the war and then acknowledged Białobrzeski's result as being earlier than his). The stability condition of the stellar interior is $L_{\star}(r)<L_{\mathrm{ed}}(r)$ where $L_{\mathrm{ed}}(r)$ is defined by Eq. (5) but with $M(r)$, the mass within a radius $r$, instead of the total mass $M$; and where the appropriate opacity for the gas is used. In the previous section we have studied the cases $L_{\mathrm{tot}}=L_{\mathrm{acc}}$ with $L_{\star}=0$, and $L_{\mathrm{tot}}=L_{\star}+L_{\mathrm{acc}}$. In Eddington's work $L_{\mathrm{tot}}=L_{\star}$ and, this should be kept in mind, $L_{\mathrm{acc}}=0$. Related to the importance of radiation pressure in 1916 , it is interesting to report a short sentence from Eddington's paper: there seems to be a rather widespread impression that gases are not subject to radiation-pressure.

The modern theory of accretion can be started with the paper by ${ }^{21}$ where it was investigated the possibility that climate variations on Earth could be induced by the increase of radiation emitted by the Sun when passing through a nebulous cloud. An explicit formula for the accretion luminosity was not given in the paper but it is clear from the text that the authors assumed Eq. (1). The effect of the radiation pressure was not taken into account, but it should be noted that for this problem $\dot{M}$ had to be small to avoid a significant increase of the Sun mass, that the authors wanted to exclude.

A similar scenario was used later to explain the presence in the Galaxy of massive stars: when it was understood that the lifetime of high-mas stars was too short in comparison with the estimated age of the universe, it was proposed by ${ }^{22}$ that accretion of matter from the interstellar medium provided the star with new hydrogen to burn (the so-called mechanism of rejuvenation). In this case the effect of the radiation pressure on accretion was studied but it was found negligible ${ }^{23}$ in case of a composition of the infalling matter consisting of fully molecular hydrogen, and interaction with the radiation only through photoexcitation. It is interesting to report this statement $b^{23}$ : It is to be noticed that there is no question of radiation pressure having effective action on the material as it approaches the surface of the star, since when the material becomes ionized radiation pressure can have no more than a negligible effect. Also the gas pressure was considered negligible, because it was assumed that any internal gain of heat would be radiated by the gas.

Later on, it was proposed by ${ }^{24}$ that the formation itself of a high-mass star was through accretion on a low-mass star moving along the ZAMS. This is an interesting hypothesis because, nowadays, it is proposed a similar mechanism to form high-mass stars (see the review by ${ }^{25}$ ).

In all cases, the framework was that of a star moving within a cloud, so that the mass accretion rate was derived as function of the mass of the star and of its velocity with respect to the cloud. The study of accretion in the opposite case, in which the gas stream is dominated not by the velocity of the accreting source but by the internal pressure of the gas (pressure-limited accretion in contrast with the velocity-limited accretion of the previous studies) was addressed by ${ }^{2}$. In the pressure-limited scenario, the velocity of the source is not important and can be set to zero, as is done nowadays assuming that the star is at rest with respect to the interstellar medium.

The effect of radiation pressure in Bondi's ${ }^{2}$ accretion model was considered by ${ }^{26}$ but taking into account only the radiation pressure due to the star luminosity, not to accretion. The conclusion was that for stars earlier than B0 radiation pressure from photoelectric absorption is not negligible.

The effect of the radiation pressure due to $L_{\text {acc }}$ on accreting matter was investigated by ${ }^{27}$ : the accretion was studied in the contest of the origin of the luminosity in quasi-stellar sources. The model was that developed by Hoyle and collaborators. The conclusion was that once a limiting value of the ratio $L / M$ is reached $\left(3 \times 10^{4}\right.$ in solar units for a pure ionized hydrogen), radiation pressure keeps $L / M$ constant. The limiting value of $L / M$ is clearly the ratio $L_{\mathrm{ed}} / M$ given by Eq. (5). After this limiting value is reached, $M$ and $L$ increase exponentially with an e-fold time $\sim 10^{9} \mathrm{yrs}$.

Another paper, not accessible through ADS, on the effects of the radiation pressure was published by Zel'dovich ${ }^{28}$. Two years later, in 1966, Zel'dovich \& Novikov ${ }^{29}$ published a long paper on relativistic astrophysics, where again this problem is addressed. In Section 5b, Zel'dovich \& Novikov derived the condition of equilibrium at the surface layer of a star through Eq. (4) with $L \equiv L_{\star}$, as in Eddington's work ${ }^{20}$. The equilibrium was found at the value of $L / M=3 \times 10^{4}$, clearly the same as in ${ }^{27}$. Later, in Section 13f, Zel'dovich \& Novikov used the same limiting value on the ratio $L / M$ to conclude that above this limit accretion is stopped, but now assuming $L \equiv L_{\text {acc }}$.

What can we conclude after this short excursus on accretion history? At the beginning, one century ago, cases under study were limited to small $\dot{M}$ for which Eq. (1) is numerically justified. The only contribution to the luminosity was $L_{\star}$ that is 
independent on accretion. Moreover, the ratio $L / M$ from Eq. (5) is very high and stars along the ZAMS do not reach it. If $L_{\mathrm{ed}}$ plays a role in star formation is because, as we have seen previously, the dust opacity is higher than Thomson opacity $\sigma / m$, up to one order of magnitude ${ }^{10}$. It was only in the ' 60 s that astronomers observed compact objects or extragalactic sources that show large ratios $L / M$, approaching the Eddington limit. As long as Eq. (4) is concerned, there is not much difference if the radiation pressure is due to accretion luminosity, stellar luminosity, or both; the equation simply states that $L_{\text {tot }}<L_{\mathrm{ed}}$ for accretion to be possible. The fundamental difference is that $L_{\text {acc }}$ depends on $\dot{M}$, while $L_{\star}$ does not (at least on time scales such that $M$ can be considered constant): as a consequence, the condition $L_{\star}<L_{\mathrm{ed}}$ has nothing to do with $\dot{M}$, and in particular with the relation between $\dot{M}$ and $L_{\mathrm{acc}}$. On the other hand, the condition $L_{\mathrm{acc}}<L_{\mathrm{ed}}$ requires to write $L_{\text {acc }}$ in terms of $\dot{M}$ and to this aim Eq. (1) was directly applied, probably based on the idea that the strength of the gravitational potential well $G M / R$ does not depend on the radiation pressure, which is by no doubt true. It was overlooked that the radiation pressure due to accretion makes work on the infalling matter, so acting as a negative feedback that self-regulates the amount of kinetic energy available at the star's surface. Even if in the '70s it was recognized that infalling matter is significantly decelerated by radiation pressure at high $\dot{M}$, this result never led to question the validity of Eq. (1).

\section{Practical applications}

\section{Burst of accretion on massive young stellar objects}

Low-mass stars $\left(M \leq 8 M_{\odot}\right)$ experience episodes of enhanced accretion that cause a burst of luminosity ${ }^{30}$, the so-called FU Orionis objects. It was not clear if a similar phenomenon is present also during the formation of high-mass stars. In the last years, three possible FU Ori candidates have been discovered; in this section I will discuss two of them, for the other one there are not enough informations on the accreting object.

\section{MM1 in G358.93-0.03}

G358.93-0.03 is a massive star-forming region where a burst has been observed from one of its sources, namely MM1. Using SOFIA observations as well as archive observations the spectral energy distribution (SED) from IR to $\mathrm{mm}^{\text {was constructed }}{ }^{31}$. By a radiative transfer analysis of the SED the following data were derived: $L_{\text {burst }}=23400 L_{\odot}$ and $L^{\text {pre }}=5000 L_{\odot}$ for the preburst luminosity; for the stellar parameters $M=12 M_{\odot}$ and $R=8.4 R_{\odot}$; the derived mass of the disk around the central source is $3.2 \times 10^{-3} M_{\odot}$. Assuming a linear decrease with time of $L_{\text {burst }}$, the luminosity comes back to $L^{\text {pre }}$ in 907 days corresponding to a release of $E_{\mathrm{acc}}=L_{\text {mean }} \Delta t=2.9 \times 10^{38} \mathrm{~J}$, where $L_{\text {mean }}$ is half of the peak luminosity.

From $E_{\text {acc }}=G M M_{\text {acc }} / R$, which comes from integration of Eq. (1) over the burst time interval, it can be derived ${ }^{31} M_{\text {acc }}=$ $5.3 \times 10^{-4} M_{\odot}$ and $\dot{M}=M_{\text {acc }} / \Delta t=3.2 \times 10^{-3} M_{\odot} \mathrm{yr}^{-1}$, where now $\Delta t$ is the duration of the accretion episode, estimated in two months, while 907 days used to compute $E_{\text {acc }}$ is the estimated time to release all the accretion luminosity. So, during the burst $16 \%$ of the disk was accreted. If confirmed, such a high value implies that these episodes cannot be very frequent or that the disk is a transient structure.

Through Eq. (20) we can derive $\dot{M}$ as

$$
\dot{M}=\frac{L_{\mathrm{tot}}-L_{\star}}{G M / R-L_{\mathrm{tot}} / \dot{M}_{\mathrm{ed}}}
$$

where $L_{\text {tot }}$ is the observed luminosity during the burst phase, while $\dot{M}$ is the total mass accretion rate, including the value during the quiescent phase that, following ${ }^{31}$, is set to 0 . Using the parameters reported above for $M$ and $R$, Eq. (27) gives $\mu=0.049$ and $\dot{M}=4.6 \times 10^{-4} M_{\odot} \mathrm{yr}^{-1}$, thus $M_{\mathrm{acc}}=7.4 \times 10^{-5} M_{\odot}$. The accreted mass is now $\sim 2 \%$ of the disk, still a not-negligible percentage but much less then the $16 \%$ value found by ${ }^{31}$.

The value of $\dot{M}$ can be further decreased if we note that the assumed $M=12 M_{\odot}$ and $R=8.4 R_{\odot}$ are valid for a ZAMS star, while this object is still accreting. For a $\dot{M}=10^{-5} M_{\odot} \mathrm{yr}^{-1}$ the model by ${ }^{32}$ predicts that a total luminosity of $5000 L_{\odot}$, of which $\sim 800 L_{\odot}$ due to accretion, is produced when $M=9.1 M_{\odot}$ and $R=3.7 R_{\odot} ;$ in the pre-burst period $\mu=0.002$ so that $L_{\text {acc }}$ does not change using Eq. (1) or Eq. (13). Now Eq. (27) gives $\dot{M}=2.5 \times 10^{-4} M_{\odot} \mathrm{yr}^{-1}$, that becomes $\dot{M}=2 \times 10^{-4} M_{\odot} \mathrm{yr}^{-1}$ if we subtract the constant $\dot{M}$ during quiescience. The total mass accreted during the burst in two months is then $M_{\text {acc }}=$ $2.5 \times 10^{-5} M_{\odot}$ or $1 \%$ of the disc mass.

\section{S255IR-SMA1}

SMA1 is a high-mass young stellar object in the massive star-forming region S255IR. The burst of NIRS3 was studied by ${ }^{33}$ who found a luminosity increase from $L^{\text {pre }}=2.9 \times 10^{4} L_{\odot}$ to $L_{\text {burst }}=1.6 \times 10^{5} L_{\odot}$ corresponding to a release of $E_{\text {acc }}=$ $1.2 \times 10^{46}$ erg over a period of 9 months; the star mass is ${ }^{34} M=20 M_{\odot}$ with a radius ${ }^{33} R=10 R_{\odot}$. These data give $\dot{M}=$ $5 \times 10^{-3} M_{\odot} \mathrm{yr}^{-1}$ and $M_{\mathrm{acc}}=3.4 \times 10^{-3} M_{\odot}$, about twice Jupiter mass.

By applying again Eq. (27) we find $\mu=0.262$ or $\dot{M}=2.9 \times 10^{-3} M_{\odot} \mathrm{yr}^{-1}$ and $M_{\text {acc }}=2.0 \times 10^{-3} M_{\odot}$, a factor 1.7 smaller than found $b^{33}$. 
However, it should be noted that a star luminosity of $2.9 \times 10^{4} L_{\odot}$ is quite low for a $20 M_{\odot}$ star for which it is suggested ${ }^{35}$ $4.1 \times 10^{4} L_{\odot}$ and $R=6 R_{\odot}$. We can then make the hypothesis, as in the previous case, that this source is still forming so that we can infer its radius from the models by ${ }^{32}$. It was derived ${ }^{36}$ for the molecular outflow of this source $\dot{M}_{\text {out }}=4 \times 10^{-4} M_{\odot} \mathrm{yr}^{-1}$; using the relation ${ }^{37} \dot{M}=\dot{M}_{\text {out }} / 6$ we derive $\dot{M}=6.7 \times 10^{-5} M_{\odot} \mathrm{yr}^{-1}$.

The mass of the central source, $20 M_{\odot}$, is constrained by the dynamics of the observed masers and is quite solid ${ }^{34}$. For this mass, however, the model md5 by ${ }^{32}$ with $\dot{M}=10^{-5} M_{\odot} \mathrm{yr}^{-1}$ predicts a much higher stellar luminosity, even without taking into account $L_{\text {acc }}: L_{\star}=4.5 \times 10^{4} L_{\odot}$. On the other hand, using also data in the (sub)mm previously published, it was derived ${ }^{34}$ $L_{\star}=3.5 \times 10^{4} L_{\odot} \cdot \dot{M}=10^{-5} M_{\odot} \mathrm{yr}^{-1}$ implies a not negligible $L_{\text {acc }}$ also during quiescent phase, so that it is assumed here that the value given by ${ }^{34}$ is $L_{\text {tot }}$ rather than $L_{\star}$. From model md5 we find the following set of parameters: $M=16 M_{\odot}$ (compatible at $2 \sigma$ level with the measured $\left.M=(20 \pm 2) M_{\odot}\right), R=5.0 R_{\odot}$ and $L_{\star}=2.5 \times 10^{4} L_{\odot}$, while Eq. $(27)$ gives $L_{\text {acc }}=9.4 \times 10^{3} L_{\odot}$. Thus, $L_{\text {tot }}=3.4 \times 10^{4} L_{\odot}$, in good agreement with the derived value of $3.5 \times 10^{4} L_{\odot}$.

Using again Eq. (27) with the data for the burst we find $\dot{M}=1.3 \times 10^{-3} M_{\odot} \mathrm{yr}^{-1}$ that becomes $1.2 \times 10^{-3} M_{\odot} \mathrm{yr}^{-1}$ after subtracting the constant $\dot{M}$. This is a factor $\sim 4.2$ smaller than the value derived by ${ }^{33}$. Consequently, $M_{\text {acc }}=8.2 \times 10^{-4} M_{\odot}$.

\section{The growth of the supermassive black holes}

An interesting application of the alternative formulae for $L_{\text {acc }}$ presented in this paper is in the field of the supermassive black holes (see the review by ${ }^{38}$ ). These black holes formed very early in the Universe reaching a mass of $\sim 10^{9} M_{\odot}$ in $\sim 10^{9}$ yrs. This implies a mean $\dot{M} \sim 1 M_{\odot} \mathrm{yr}^{-1}$ that even for a seed of $100 M_{\odot}$ is such that, from Eq. (22), $\dot{M} \gg \dot{M}_{\mathrm{ed}}$.

Such high rates represent a big challenge to the standard theory of accretion where, when $\mu=1$, i.e., $\dot{M}=\dot{M}_{\text {ed }}$, accretion is stopped by radiation pressure. The solution to the problem is found in particular accretion geometry, or looking for radiatively inefficient accretion flows (RIAF, see previous section). On the contrary, $L_{\text {acc }}$ derived with Eq. (14) can support any accretion rate, also with $\mu>1$.

Suppose that a SMBH starts with a mass of $100 M_{\odot}$ and accretes with a constant $\dot{M}=1 M_{\odot} \mathrm{yr}^{-1}$, so that the mass grows linearly with time. In the top panel of Fig. 3, $M, L_{\mathrm{acc}}, L_{\mathrm{ed}}$ and $\mu=\dot{M} / \dot{M}_{\mathrm{ed}}$ are shown as function of time. At the start of accretion $\mu$ is very high, $2.2 \times 10^{6}$, because $\dot{M}_{\text {ed }}$ is small, see Eq. (22). As a consequence, $L_{\text {acc }} \sim L_{\text {ed }} \sim 3.3 \times 10^{6} L_{\odot}$. After $10^{9} \mathrm{yrs}, L_{\mathrm{ed}}=3.3 \times 10^{13} L_{\odot}$ while $L_{\mathrm{acc}}=6 \times 10^{12} L_{\odot}$, and $\mu=0.2$.

In the bottom panel of Fig. 3 the same curves are shown but assuming $\dot{M}$ varying with time as ${ }^{38} \dot{M}=4 \times 10^{-3} M_{\odot} \mathrm{yr}^{-1} T_{3}^{3 / 2}$, where $T_{3}$ is time in $10^{3}$ yrs. $\dot{M}$ is very low at the beginning, $1.3 \times 10^{-7} M_{\odot} \mathrm{yr}^{-1}$, increasing up to $5 \times 10^{2} M_{\odot} \mathrm{yr}^{-1}$ at the end of accretion. The initial mass of the black hole is again $100 M_{\odot}$ and the final mass of $10^{9} M_{\odot}$ is attained after $2.5 \times 10^{6}$ yrs, about $1 / 1000$ of the previous case. $L_{\mathrm{ed}}$ is the same as in the previous case because initial and final mass do not change. $L_{\text {acc }}$ starts with the value $7.2 \times 10^{5} L_{\odot}$ approaching $L_{\mathrm{ed}}$ as $\dot{M}$ and $\mu$ increase. $\mu$ is high already at the beginning of the accretion, with value 0.28 , and reaches its maximum of $3.1 \times 10^{4}$ after $\sim 10^{3}$ yrs; afterwards, the increase in $\dot{M}_{\text {ed }}$ is higher than that of $\dot{M}$ so that $\mu$ decreases to 88 , in any case larger than 1 .

\section{Conclusions}

As written in the Introduction, the aim of this paper is to suggest that when modelling accretion onto an object, being a star or a black hole, instead of assuming that $L_{\mathrm{acc}}=G M \dot{M} / R$, Eq. (1), as universally done, one should use $L_{\mathrm{acc}}=v_{\mathrm{f}}^{2} \dot{M} / 2$, Eq. (2), where $v_{\mathrm{f}}$ is the velocity of the infalling matter when hits the surface of the accreting object.

The two formulations of $L_{\text {acc }}$ are equivalent when the radiation pressure only due to accretion is negligible. Both are based on energy conservation, but the former is valid when matter is in free fall, while the latter holds always.

For the simple case of spherical accretion of pure ionized hydrogen when absorption of radiation is not considered, I showed what are the implications of using Eq. (2): $L_{\text {acc }}$ is always smaller than $L_{\mathrm{ed}}$ no matter how high $\dot{M}$ is. $L_{\mathrm{ed}}$ is reached asymptotically as $\dot{M} \rightarrow \infty$. This is a consequence of the negative feedback that the radiation pressure exerts on the infalling matter: the velocity is reduced and there is less kinetic energy to be extracted and radiated. The decrease in kinetic energy reflects the work done by radiation pressure to slow down the infalling matter. An increase in $\dot{M}$ would imply a linear increase in $L_{\text {acc }}$, but this increase is in part compensated by the breaking work of the radiation, so that the effective increase is not linear at high $\dot{M}$. This work is not accounted for when Eq. (1) is used. As long as Eq. (1) is assumed valid a-priori, one will derive that $L_{\text {acc }}=L_{\text {ed }}$ for some $\dot{M}$.

If the accreting object emits by its own radiation at a rate $L_{\star}$, for example due to thermonuclear reactions, then accretion is still possible if $L_{\star}<L_{\mathrm{ed}}$. In this case too $L_{\mathrm{acc}}$ will always be less than $L_{\mathrm{ed}}$ or, better, less than $\left(L_{\mathrm{ed}}-L_{\star}\right)$. When $L_{\star}>L_{\mathrm{ed}}$, not only accretion is no longer possible (as long as there are no other mechanisms that can push matter), but increasing $\dot{M}$ has the counterintuitive effect of reducing the luminosity from the system as seen by an observer. This simply reflects the work done by radiation pressure.

I have applied the formulae found in this paper in a few cases: the formation of high-mass stars, and the growth of super massive black holes. Especially the latter example seems to me impressive. 

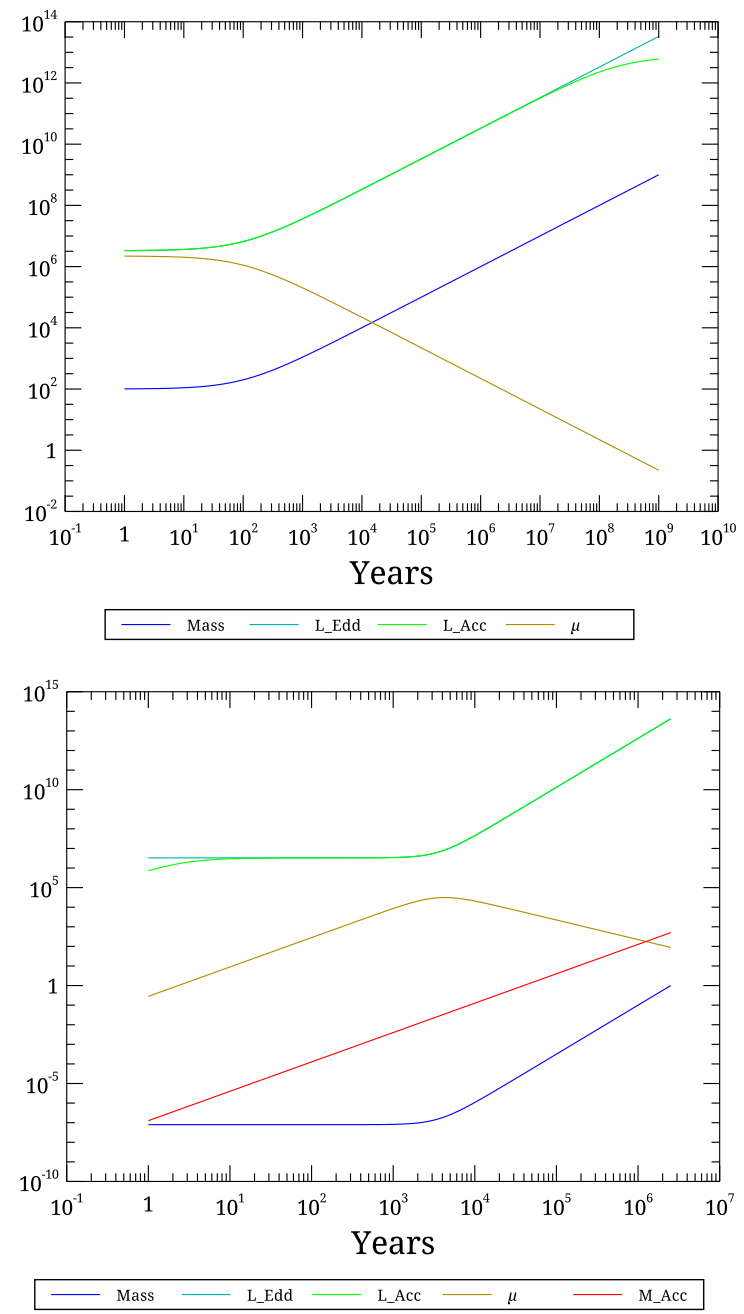

Figure 3. Top panel: mass $M, L_{\mathrm{ed}}, L_{\mathrm{acc}}, \mu=\dot{M} / \dot{M}_{\mathrm{ed}}, \dot{M}_{\mathrm{ed}}$, vs. time for a black hole of initial mass $1 M_{\odot}$ accreting at a constant $\dot{M}$. Bottom panel: same curves in case of $\dot{M}$ varying with time as $t^{3 / 2}$. Mass and luminosities are in solar units; $\mu=\dot{M} / \dot{M}_{\text {ed }}$ is adimensional. In the bottom panel, M_Acc is $\dot{M}$ in $M_{\odot} \mathrm{yr}^{-1}$. 
From the historical reconstruction presented in the text, I suggest that the reason why Eq. (1) has never been questioned is because at the origin small $\dot{M}$ were considered and often the radiation was that generated by the star rather than produced by accretion. At a certain moment, the conclusion that $L_{\star}$ can halt accretion, that is certainly true, was applied directly to $L_{\text {acc }}$ : but while $L_{\star}$ does not depend on accretion, so that any influence of the radiation pressure on the motion of the gas cannot change the internal luminosity of the star, on the other hand $L_{\text {acc }}$ does depend on the motion, and in particular, on the kinetic energy acquired by matter in its falling toward the central source.

\section{References}

1. Frank, J., King, A. R. \& Raine, D. J. Accretion power in astrophysics (Cambridge Astrophysics Series, Cambridge: University Press, 1985, 1985).

2. Bondi, H. On spherically symmetrical accretion. Mon. Notices Royal Astron. Soc. 112, 195, DOI: 10.1093/mnras/112.2.195 (1952).

3. Landau, L. D. \& Lifshitz, E. M. The classical theory of fields (Oxford: Pergamon Press, 1971, 1971).

4. Maraschi, L., Reina, C. \& Treves, A. On Spherical Accretion near the Eddington Luminosity. Astron. Astrophys. 35, 389 (1974).

5. Eckart, A., Schödel, R. \& Straubmeier, C. The black hole at the center of the Milky Way (London:Imperial College Press, 2005).

6. Degregoria, A. J. An Investigation of Accretion of Matter onto White Dwarfs as a Possible X-Ray Mechanism. Astrophys. J. 189, 555-562, DOI: 10.1086/152834 (1974).

7. Kahn, F. D. Cocoons around early-type stars. Astron. Astrophys. 37, 149-162 (1974).

8. Wolfire, M. G. \& Cassinelli, J. P. Conditions for the Formation of Massive Stars. Astrophys. J. 319, 850, DOI: $10.1086 / 165503$ (1987).

9. McKee, C. F. \& Ostriker, E. C. Theory of Star Formation. Annu. Rev. Astron. Astrophys. 45, 565-687, DOI: 10.1146/annurev.astro.45.051806.110602 (2007). 0707.3514.

10. Pollack, J. B. et al. Composition and Radiative Properties of Grains in Molecular Clouds and Accretion Disks. Astrophys. J. 421, 615, DOI: 10.1086/173677 (1994).

11. Shapiro, S. L. Accretion onto Black Holes: the Emergent Radiation Spectrum. Astrophys. J. 180, 531-546, DOI: 10.1086/151982 (1973).

12. Begelman, M. C. Can a spherically accreting black hole radiate very near the Eddington limit? Mon. Notices Royal Astron. Soc. 187, 237-251, DOI: 10.1093/mnras/187.2.237 (1979).

13. Taam, R. E., Fu, A. \& Fryxell, B. A. Accretion in Wind-driven X-Ray Sources. Astrophys. J. 371, 696, DOI: $10.1086 / 169935$ (1991).

14. Burger, H. L. \& Katz, J. I. The Eddington limit and supercritical accretion. I - Time-independent calculations. Astrophys. J. 236, 921-927, DOI: 10.1086/157819 (1980).

15. Yuan, F., Quataert, E. \& Narayan, R. Nonthermal Electrons in Radiatively Inefficient Accretion Flow Models of Sagittarius A*. Astrophys. J. 598, 301-312, DOI: 10.1086/378716 (2003). astro-ph/0304125.

16. Maraschi, L., Reina, C. \& Treves, A. The effect of radiation pressure on accretion disks around black holes. Astrophys. J. 206, 295-300, DOI: 10.1086/154384 (1976).

17. Fukue, J. Bondi Accretion onto a Luminous Object. Publ. Astrophys. Soc. Jpn. 53, 687-692, DOI: 10.1093/pasj/53.4.687 (2001).

18. Omukai, K. \& Palla, F. Formation of the First Stars by Accretion. Astrophys. J. 589, 677-687, DOI: 10.1086/374810 (2003). astro-ph/0302345.

19. Abramowicz, M. A. Super-Eddington black hole accretion: Polish doughnuts and slim disks. In Merloni, A., Nayakshin, S. \& Sunyaev, R. A. (eds.) Growing Black Holes: Accretion in a Cosmological Context, 257-273, DOI: 10.1007/11403913_49(2005). astro-ph/0411185.

20. Eddington, A. S. On the radiative equilibrium of the stars. Mon. Notices Royal Astron. Soc. 77, 16-35, DOI: 10.1093/mnras/77.1.16 (1916).

21. Hoyle, F. \& Lyttleton, R. A. The effect of interstellar matter on climatic variation. Math. Proc. Camb. Philos. Soc. 35, 405, DOI: 10.1017/S0305004100021150(1939). 
22. Lyttleton, R. A. \& Hoyle, F. The evolution of the stars. The Obs. 63, 39-43 (1940).

23. Hoyle, F. \& Lyttleton, R. A. On the physical aspects of accretion by stars. Math. Proc. Camb. Philos. Soc. 36, 424, DOI: $10.1017 / \mathrm{S} 0305004100017461$ (1940).

24. Bondi, H. \& Hoyle, F. On the mechanism of accretion by stars. Mon. Notices Royal Astron. Soc. 104, 273, DOI: 10.1093/mnras/104.5.273 (1944).

25. Zinnecker, H. \& Yorke, H. W. Toward Understanding Massive Star Formation. Annu. Rev. Astron. Astrophys. 45, 481-563, DOI: 10.1146/annurev.astro.44.051905.092549(2007). 0707.1279.

26. Mestel, L. The influence of stellar radiation on the rate of accretion. Mon. Notices Royal Astron. Soc. 114, 437, DOI: 10.1093/mnras/114.4.437 (1954).

27. Salpeter, E. E. Accretion of Interstellar Matter by Massive Objects. Astrophys. J. 140, 796-800, DOI: 10.1086/147973 (1964).

28. Zel'dovich, Y. B. The Fate of a Star and the Evolution of Gravitational Energy Upon Accretion. Sov. Phys. Doklady 9, 195 (1964).

29. Zel'dovich, Y. B. \& Novikov, I. D. Reviews of Topical Problems: Relativistic Astrophysics. Ii. Sov. Phys. Uspekhi 8, 522-577, DOI: 10.1070/PU1966v008n04ABEH002990 (1966).

30. Hartmann, L. \& Kenyon, S. J. On the nature of FU Orionis objects. Astrophys. J. 299, 462-478, DOI: 10.1086/163713 (1985).

31. Stecklum, B. et al. Infrared observations of the flaring maser source G358.93-0.03. SOFIA confirms an accretion burst from a massive young stellar object. Astron. Astrophys. 646, A161, DOI: 10.1051/0004-6361/202039645 (2021). 2101.01812.

32. Hosokawa, T. \& Omukai, K. Evolution of Massive Protostars with High Accretion Rates. Astrophys. J. 691, 823-846, DOI: $10.1088 / 0004-637 X / 691 / 1 / 823$ (2009). 0806.4122.

33. Caratti o Garatti, A. et al. Disk-mediated accretion burst in a high-mass young stellar object. Nat. Phys. 13, 276-279, DOI: 10.1038/nphys3942 (2017). 1704.02628.

34. Zinchenko, I. et al. The Disk-outflow System in the S255IR Area of High-mass Star Formation. Astrophys. J. 810, 10, DOI: $10.1088 / 0004-637 X / 810 / 1 / 10$ (2015). 1507.05642.

35. Davies, B. et al. The Red MSX Source survey: critical tests of accretion models for the formation of massive stars. Mon. Notices Royal Astron. Soc. 416, 972-990, DOI: 10.1111/j.1365-2966.2011.19095.x (2011). 1105.3984.

36. Wang, Y. et al. Different evolutionary stages in the massive star-forming region $\mathrm{S} 255$ complex. Astron. Astrophys. 527, A32, DOI: 10.1051/0004-6361/201015543 (2011). 1011.3575.

37. Beltrán, M. T. \& de Wit, W. J. Accretion disks in luminous young stellar objects. Astron. Astrophys. 24, 6, DOI: 10.1007/s00159-015-0089-z (2016). 1509.08335.

38. Inayoshi, K., Visbal, E. \& Haiman, Z. The Assembly of the First Massive Black Holes. Annu. Rev. Astron. Astrophys. 58, 27-97, DOI: 10.1146/annurev-astro-120419-014455 (2020). 1911.05791.

\section{Acknowledgements}

I thank Prof. Takashi Hosokawa for providing me the URL to download the results of the models by ${ }^{32}$.

This research has made use of: NASA's Astrophysics Data System Bibliographic Services; the SIMBAD database, operated at CDS, Strasbourg, France.

\section{Additional information}

Competing interests: the author declares no competing interests. 